\title{
La codificación CIE-10 en la enfermedad pulmonar intersticial en el Instituto Nacional de Enfermedades Respiratorias (INER) ¿sigue siendo aplicable?
}

\author{
José Iván Betanzos-Paz, Mayra Edith Mejía-Ávila, Ivette Buendía-Roldán
}

Instituto Nacional de Enfermedades Respiratorias Ismael Cosío Villegas, Ciudad de México.

Trabajo recibido: 19-V-2016; aceptado: 14-IX-2016

\begin{abstract}
RESUMEN. Antecedentes: Las enfermedades pulmonares intersticiales (EPI) son un grupo heterogéneo de enfermedades que afectan al intersticio pulmonar. Material y métodos: Se realizó un estudio observacional, retrospectivo y descriptivo de los pacientes egresados de enero de 2010 a junio de 2015, con los códigos CIE-10: (J67) neumonitis hipersensitiva debida a polvo orgánico, (J82) eosinofilia pulmonar no clasificada en otra parte, (J84) otras enfermedades pulmonares intersticiales; y los diferentes numerales disponibles en la clasificación para cada uno de ellos para corroborar si la codificación comprendía al total de diagnósticos emitidos en el Servicio de Enfermedades Intersticiales del Instituto. Resultados: Se evaluaron 1,279 pacientes egresados, clasificándolos de acuerdo al diagnóstico del médico tratante, resultando los más frecuentes: EPI, 538 (42\%); NH, 223 (17.4\%); FPI, 185 (14.4\%); fibrosis pulmonar no clasificable (FPNC), 155 (12.1\%) y NINE, 95 (7.4\%); mientras que el CIE-10 los agrupó en forma indistinta. Conclusiones: Con los resultados obtenidos en este trabajo corroboramos que la codificación CIE-10 para enfermedades intersticiales es insuficiente para la heterogeneidad de diagnósticos y se debe unificar para poder realizar estudios epidemiológicos en un futuro.
\end{abstract}

Palabras clave: CIE-10, enfermedad pulmonar intersticial, codificación.

ABSTRACT. Background: Interstitial lung disease (ILD) is a heterogeneous group of diseases that affects pulmonary interstitium. Material and methods: An observational, retrospective and descriptive study was conducted in patients discharged from January 2010 to June 2015 , with the codes ICD-10: (J67) hypersensitivity pneumonitis, (J82) eosinophilia unclassified, (J84) other interstitial lung diseases, and the different numerals available for each of them. To evaluate if clinical diagnosis reported in the interstitial lung diseases service were similar to ICD coding used. Results: We included 1,279 patients, classifying them according to the physician's diagnosis, the most common diseases reported was: ILD 538 (42\%), HP 223 (17.4\%), IPF 185 (14.4\%), unclassifiable pulmonary fibrosis (UPF) 155 (12.1\%) and NSIP 95 (7.4\%), while the ICD-10 grouped them indistinctly. Conclusion: The results obtained in this study corroborated that ICD-10 code using in ILD is insufficient for all the diagnoses included in this group so it is necessary unify the codes to perform epidemiological studies in the future.

Key words: ICD-10, interstitial lung disease, coding.

\section{INTRODUCCIÓN}

La enfermedad pulmonar intersticial (EPI) engloba a una amplia variedad de entidades heterogéneas cuya característica común es que afectan principalmente al intersticio pulmonar, este grupo de enfermedades incluye alrededor de 150 patologías diversas, como son: las diferentes neumonías intersticiales idiopáticas (NII) especialmente a la fibrosis pulmonar idiopática (FPI), la neumonitis por hipersensibilidad $(\mathrm{NH})$ y las EPI secundarias a enfermedades del tejido conectivo (EPI-ETC) entre otras. ${ }^{1}$

La incidencia y prevalencia de las diferentes EPI se desconocen en forma precisa debido a la diversidad de los datos reportados en la literatura así como a los datos epidemiológicos disponibles en los diferentes países, por ejemplo la FPI, que se considera la forma más común de las NII tiene una incidencia de 0.6 a 17.4 casos/100,000 habitantes/año y una prevalencia de 0.7 a 63 casos/100,000 habitantes, según la serie que se revise, ${ }^{2}$ mientras que la $\mathrm{NH}$, que es otra entidad frecuente en nuestro medio, tiene una incidencia de 1 a 4 casos/100,000 habitantes/año. ${ }^{3}$ En México, no contamos con datos epidemiológicos precisos en muestra poblacional, por lo que únicamente podemos referir un estudio realizado en el Instituto Nacional de Enfermedades Respiratorias Ismael Cosío Villegas 
(INER) de la Ciudad de México, en un período de 10 años (2000 a 2010) donde se observó un incremento en la morbimortalidad de la EPI, lo que se atribuye a un diagnóstico más eficiente y la notificación de estas patologías en nuestro país. ${ }^{4}$

Por otro lado, existen otros grupos de EPI que se asocian con exposición a polvos inorgánicos como carbón, asbesto y sílice, pero estas enfermedades no se diagnostican en el INER debido a su carácter laboral. Sin embargo, en los últimos años se ha reportado un incremento en el diagnóstico de la EPI secundaria a enfermedades autoinmunes (artritis reumatoide, esclerosis sistémica, dermatopolimiositis, enfermedad mixta del tejido conectivo y lupus eritematoso sistémico) debido al uso sistemático de perfiles reumatológicos disponibles en la actualidad en nuestro laboratorio. 5,6

El INER al ser un centro de referencia nacional para el diagnóstico y manejo de las enfermedades pulmonares cuenta con un gran número de pacientes con EPI, razón de interés para conocer los diagnósticos emitidos de los pacientes egresados de la Clínica de Enfermedades Pulmonares Intersticiales en los últimos años y si éstos tienen una correspondencia de acuerdo con la Clasificación Internacional de Enfermedades en su décima versión (CIE-10), información que representaría una herramienta de gran utilidad para los reportes epidemiológicos necesarios en nuestro país.

\section{MATERIAL Y MÉTODOS}

Se realizó un estudio observacional, retrospectivo y descriptivo con el Departamento de Bioestadística y la Clínica de Enfermedades Intersticiales del Pulmón del INER, se registraron los diagnósticos emitidos por el médico tratante de los pacientes egresados en el período de tiempo comprendido de enero de 2010 a junio de 2015. Asimismo, se reviso el código CIE-10 registrado en la hoja de egreso hospitalario relacionados con las enfermedades respiratorias que afectan el intersticio pulmonar como son: (J67) neumonitis hipersensitiva debida a polvo orgánico, (J82) eosinofilia pulmonar no clasificada en otra parte, (J84) otras enfermedades pulmonares intersticiales, y los diferentes numerales disponibles en la clasificación para cada uno de ellos. ${ }^{7}$

A partir de la base de datos proporcionada por bioestadística y revisión de las altas hospitalarias se separaron a los pacientes por año de egreso y diagnóstico final emitido, siendo agrupados en: neumonía intersticial no específica (NINE), EPI, NH, neumonía organizada criptogénica (NOC), proteinosis alveolar pulmonar (PAP), EPI-ETC, fibrosis pulmonar no clasificable (FPNC), fibrosis pulmonar familiar (FPF), fibrosis pulmonar avanzada (FPA), FPI y enfermedades misceláneas (EM). Los resultados fueron registrados y analizados con el programa Excel 14.6.3.

\section{RESULTADOS}

Se identificaron 1,279 casos egresados en el período de tiempo seleccionado para este estudio. Al revisar el diagnóstico de egreso emitido por la Clínica de Enfermedades Intersticiales, la distribución de los mismos fue de la siguiente manera: 538 fueron $\mathrm{EPI}(42 \%), 223$ fueron $\mathrm{NH}$ (17.4\%), 185 fueron FPI (14.4\%), 155 fueron FPNC (12.1\%), 95 fueron NINE (7.4\%), 27 fueron FPA (2\%), 17 fueron EPI-ETC (1.3\%), 16 fueron PAP (1.2\%), 14 fueron NOC (1\%), 6 fueron FPF (0.4\%), y 3 fueron EM $(0.2 \%)$ (tabla 1$)$.

Después de este primer análisis, al detectar que 538 $(42 \%)$ casos contaban con diagnóstico de egreso de EPI, se decidió revisar de forma multidisciplinaria cada uno de estos expedientes, con lo cual se pudo concretar un diagnóstico preciso sólo en 92 casos (17\%), los cuales fueron distribuidos de la siguiente manera: 47/92, EPI-ETC (51\%); 12/92, NH (13\%); 7/92, NINE (8\%); 7/92, FPA (8\%); 6/92, FPNC (7\%); 4/92, FPI (4\%); $3 / 92$ neumonía intersticial descamativa, (3\%); 2/92, NOC (2\%); $1 / 92$, angiomatosis (1\%); $1 / 92$, bronquiolitis (1\%); $1 / 92$, enfisema combinado con fibrosis pulmonar (1\%); y $1 / 92$, fibrosis intersticial centrada a la vía aérea, (1\%). Sin embargo, en los restantes 446 (83\%) casos del grupo de EPI no se logró reclasificar para un diagnóstico más específico con los datos disponibles.

De acuerdo con los códigos CIE-10 que corresponden a enfermedades respiratorias principalmente afectando al intersticio, observamos que de los 1,279

Tabla 1. Diagnósticos del Servicio Clínico 1 del INER 2010-2015.

\begin{tabular}{lrl}
\hline Diagnóstico de EPI & $\mathrm{n}=1,279(\%)$ \\
\hline Enfermedad pulmonar intersticial & $538(42)$ \\
Neumonitis por hipersensibilidad & $223(17.4)$ \\
Fibrosis pulmonar idiopática & $185(14.4)$ \\
Fibrosis pulmonar no clasificable & $155(12.1)$ \\
Neumonía intersticial no específica & $95(7.4)$ \\
EPI secundaria a enfermedad del tejido & $17(1.3)$ \\
conectivo & $27(2)$ \\
Fibrosis pulmonar avanzada & $14(1)$ \\
Neumonía organizada criptogénica & $16(1.2)$ \\
Proteinosis alveolar pulmonar & $6(0.4)$ \\
Fibrosis pulmonar familiar & $3(0.2)$ \\
Misceláneos (ACIF, NID, bronquiolitis, & \\
angiomatosis, enfisema combinado con & \\
fibrosis) & \\
\hline
\end{tabular}

ACIF = Fibrosis intersticial centrada a la vía aérea; NID = Neumonía intersticial descamativa. 
casos estudiados éstos se distribuían de la siguiente manera: $n=13$ (J84.0) condiciones alveolares y parietoalveolares; $n=396$ (J84.1) otras enfermedades pulmonares intersticiales con fibrosis; $n=652$ (J84.9) enfermedad pulmonar intersticial sin especificar; $n=205$ (J67) neumonitis por hipersensibilidad; y $\mathrm{n}=13$ (J44.8) bronquiolitis obliterante (tabla 2).

Cuando se compararon los diagnósticos emitidos por los médicos del Servicio de Enfermedades Intersticiales y los códigos asignados de acuerdo al CIE-10 pudo apreciarse que la codificación internacional de las diferentes EPI tiende a agruparlas en forma indistinta, aun cuando varias de estas entidades clasificadas bajo un mismo rubro presentan un comportamiento clínico, tomográfico e histopatológico diferente, e incluso el impacto pronóstico y de supervivencia está bien descrito para cada una de ellas (tabla 3).

\section{DISCUSIÓN}

El CIE-10 es el acrónimo de la Clasificación Internacional de Enfermedades en su décima versión, proviene

Tabla 2. Diagnósticos de acuerdo con CIE-10, Servicio de Bioestadística del INER 2010-2015.

\begin{tabular}{lc}
\hline Diagnóstico CIE-10 & $\mathrm{n}=1,279(\%)$ \\
\hline $\begin{array}{l}\text { J84.0 Condiciones alveolares y parietoal- } \\
\text { veolares }\end{array}$ & $13(1)$ \\
$\begin{array}{l}\text { J84.1 Otras enfermedades pulmonares } \\
\text { intersticiales con fibrosis }\end{array}$ & $396(31)$ \\
J84.9 Enfermedad pulmonar intersticial & $652(51)$ \\
sin especificar & \\
J67.9 Neumonitis por hipersensibilidad & $205(16)$ \\
J44.8 Bronquiolitis obliterante & $13(1)$ \\
\hline
\end{tabular}

Tabla 3. Comparación entre clasificación CIE-10 vs. diagnósticos del Servicio Clínico 1 del INER.

\begin{tabular}{|c|c|c|c|}
\hline J84.0 & $13(1)$ & Proteinosis & $16(1.2)$ \\
\hline J84.1 & $396(31)$ & $\begin{array}{l}\text { FPI } \\
\text { Fibrosis pulmonar } \\
\text { No clasificable }\end{array}$ & $373(29.1)$ \\
\hline J84.9 & $652(51)$ & $\begin{array}{l}\text { Familiar } \\
\text { NINE } \\
\text { EPI-ETC } \\
\text { Misceláneos } \\
\text { EPI* }^{*}\end{array}$ & $653(51.3)$ \\
\hline J67.9 & $205(16)$ & $\begin{array}{l}\text { Neumonitis por } \\
\text { hipersensibilidad }\end{array}$ & $223(17.4)$ \\
\hline J44.8 & $13(1)$ & NOC & $14(1)$ \\
\hline
\end{tabular}

*Antes de reevaluar los diagnósticos por equipo multidisciplinario. del inglés International Statistical Classification of Diseases and Related Health Problems, determina la clasificación y codificación de las enfermedades así como una amplia variedad de signos, síntomas, hallazgos anormales, denuncias, circunstancias sociales y causas externas de daños y/o enfermedad.

El propósito del CIE es permitir un almacenamiento y análisis sistemático, interpretación y comparación de mortalidad y morbilidad de los datos recolectados de diferentes países alrededor del mundo. Se utiliza para traducir diagnósticos a códigos alfanuméricos y realizar un análisis y almacenamiento de manera más sencilla.

En la práctica clínica, el CIE se ha vuelto una herramienta de clasificación diagnóstica en el ámbito epidemiológico y se utiliza para el monitoreo de la incidencia y prevalencia de diferentes enfermedades. Es importante resaltar que aunque se diseñó para la clasificación de enfermedades con un diagnóstico formal, no todos los problemas o razones por los cuales se acude con un proveedor de servicios médicos puede ser categorizado. ${ }^{8}$

Dentro de sus debilidades está que no tiene por separado los diferentes diagnósticos del amplio y heterogéneo grupo de las enfermedades pulmonares intersticiales, lo que genera un subdiagnóstico que dificulta la realización de estudios epidemiológicos adecuados en nuestro país.

Cuando se revisa el CIE-10 en el capítulo de enfermedades del sistema respiratorio nos enfrentamos con el hecho que éste sólo cuenta con los siguientes códigos para las EPI: (J67) neumonitis hipersensitiva debida a polvo orgánico, (J67.0) pulmón de granjero, (J67.1) bagazosis, (J67.2) pulmón de colombófilo, (J67.3) suberosis, (J67.4) pulmón de recolector de malta, (J67.5) pulmón de recogedor de setas, (J67.6) pulmón de recipiente de corteza de arce, (J67.7) pulmón de aire acondicionado y humidificador, (J67.8) neumonitis hipersensitiva debida a otro tipo de polvo orgánico, (J67.9) neumonitis hipersensitiva debida a otro tipo de polvo orgánico sin especificar, (J82) eosinofilia pulmonar no clasificada en otra parte, (J84) otras enfermedades pulmonares intersticiales, (J84.0) condiciones alveolares y parietoalveolares, (J84.1) otras enfermedades pulmonares intersticiales con fibrosis, (J84.8) otras enfermedades pulmonares intersticiales especificadas y (J84.9) enfermedad pulmonar intersticial sin especificar.

En relación con los diagnósticos de las EPI que detectamos a partir de los datos del Servicio de Bioestadística a través de los códigos del CIE-10, identificamos que el grupo de las NII que ha sido ampliamente evaluado y considera ocho entidades bien caracterizadas, ${ }^{9}$ no cuenta con un código específico para cada una de ellas. Un ejemplo es la NINE, la cual 
representa cerca del $10 \%$ de los diagnósticos emitidos en nuestro centro y no cuenta con un código específico CIE-10, por lo que podría ser clasificada de acuerdo a si es inflamatoria o fibrótica en alguno de los siguientes rubros: otras enfermedades pulmonares intersticiales con fibrosis (J84.1); o bien en el de otras enfermedades pulmonares intersticiales especificadas (J84.9), siendo que de acuerdo con la decisión de cada codificador nos modificaría la frecuencia de la misma y la interpretación de los datos solicitados con fines epidemiológicos. Pero aún de mayor trascendencia está el caso de la FPI, la cual representa el prototipo de las enfermedades fibrosantes idiopáticas crónicas y progresivas, que de acuerdo con la literatura abarca cerca del $20 \%$ de todos los casos de EPI ${ }^{7,9,10}$ y la cual tampoco tiene un código específico para ser clasificada, en este caso se codifica en el rubro otras enfermedades pulmonares intersticiales con fibrosis (J84.1) junto con entidades intersticiales de un pronóstico diferente; siendo en el momento actual en todos los países una información requerida de suma importancia a partir de la aprobación de nuevos medicamentos específicos para el uso exclusivo en FPI.

Otro dato observado y relevante en nuestro estudio es el elevado número de pacientes con diagnóstico de egreso de EPI, siendo de 538 pacientes para este período de evaluación (enero 2010 a junio 2015). Consideramos que esto puede deberse a que el continuo entendimiento de la patología intersticial ha tenido una modificación constante a partir de los diferentes consensos de expertos a nivel internacional utilizando criterios diagnósticos unificados para este tipo de patologías; un ejemplo de ello es el grupo de entidades intersticiales que se han asociado con enfermedad autoinmune no caracterizada y que recién se clasifica como una neumonía intersticial con características autoinmunes (IPAF) término propuesto por la ERS/ATS Task Force on Undifferentiated Forms of CTD-ILD.11,12

Los pacientes con IPAF tienen características de EPI y datos subclínicos de ETC, estos pacientes en general tienen un curso clínico más favorable que aquéllos con FPI; aunque lo más relevante es que se les puede ofrecer tratamiento inmunosupresor y antiinflamatorio lo que mejora su pronóstico. ${ }^{11}$ Por esta razón se ha propuesto unificar criterios para confirmar el diagnóstico de los pacientes, la nueva clasificación incorpora no sólo manifestaciones serológicas o clínicas de ETC; sino, además, características morfológicas sugestivas de ETC en la tomografía computada de alta resolución, biopsia pulmonar quirúrgica y pruebas de función pulmonar. ${ }^{12}$

Otro objetivo por realizar es una correcta clasificación de las EPI pues las estadísticas mexicanas son nulas. Al revisar la base de datos del INEGI podemos observar que sólo existen estadísticas de la enfermedad pulmonar obstructiva crónica y no existe ningún dato acerca de las EPI, por lo que la información obtenida en este estudio crea un precedente para resaltar la importancia de los diagnósticos adecuados en un centro de referencia, en donde estas entidades en forma global están dentro de las primeras cinco causas de morbimortalidad.

Así, consideramos que si se planteara una modificación a la clasificación del CIE-10 en los diagnósticos de EPI se podría incrementar los estudios epidemiológicos en nuestro país y contribuir con los estudios de registros de las diferentes entidades englobados en la patología intersticial. Información que cada vez, con mayor frecuencia, es requerida a nivel internacional.

\section{CONCLUSIONES}

La codificación de CIE-10 actual para las EPI es insuficiente para englobar la gama de entidades consideradas bajo este término. El modificar esta codificación tomando en cuenta los consensos internacionales en enfermedades intersticiales permitiría realizar estudios epidemiológicos verídicos en un futuro, mismos que son de suma importancia para la incorporación de nuevas moléculas terapéuticas en las diferentes entidades. Además, utilizar una clasificación más específica permitirá tomar decisiones para mejorar las políticas de salud en este grupo de enfermedades del país.

\section{Agradecimientos}

Al Departamento de Bioestadística del INER por la búsqueda de diagnósticos solicitada y al Dr. Jorge Valdés por la captura parcial de la información en la base de datos.

\section{REFERENCIAS}

1. Marcos PJ, Montero C, Otéro GI. Una mirada general a las enfermedades pulmonares intersticiales y una específica a la fibrosis pulmonar idiopática. Galicia Clin 2013;74(1):13-22.

2. Ley B, Collard HR. Epidemiology of idiopathic pulmonary fibrosis. Clin Epidemiol 2013;5:483-492. doi 10.2147/ CLEP. S54815.

3. Selman M, Pardo A, King TE Jr. Hypersensitivity pneumonitis: insights in diagnosis and pathobiology. Am J Respir Crit Care Med 2012;186(4):314-324. doi: 10.1164/ rccm.201203-0513Cl.

4. Martínez-Briseño D, García-Sancho C, Fernández-Plata R, Franco-Marina F, Torre-Bouscoulet L, Pérez-Padilla R. Tendencia de la mortalidad por enfermedades intersti- 
ciales en México, período 2000-2010. Neumol Cir Torax 2014;73(3):179-184.

5. Pérez-Dórame R, Mejía M, Mateos-Toledo H, RojasSerrano J. Rheumatoid arthritis-associated interstitial lung disease: lung inflammation evaluated with high resolution computed tomography scan is correlated to rheumatoid arthritis disease activity. Reumatol Clin 2015;11(1):12-16. doi: 10.1016/j.reuma.2014.02.007.

6. Rojas-Serrano J, González-Velásquez E, Mejía M, Sánchez-Rodríguez A, Carrillo G. Interstitial lung disease related to rheumatoid arthritis: evolution after treatment. Reumatol Clin 2012;8(2):68-71. doi: 10.1016/j. reuma.2011.12.008.

7. WHO. Classifications. International classification of diseases (ICD). Anexo: CIE-10. Enfermedades del sistema respiratorio. Fecha de consulta: 29-06-2014. Accesible en: www.who.int/whosis/icd10/

8. International Statistical Classification of Diseases and Related Health Problems. 10th Revision. Accessible en: www.who.int/classifications/icd/ICD10Volume2_ en_2010.pdf

9. Travis WD, Costabel U, Hansell DM, etal.; ATS/ERS Committee on Idiopathic Interstitial Pneumonias. An official American Thoracic Society/European Respiratory Society statement: update of the international multidisciplinary classification of the idiopathic interstitial pneumonias.
Am J Respir Crit Care Med 2013;188(6):733-748. doi: 10.1164/rccm.201308-1483ST.

10. Sgalla G, Biffi A, Richeldi L. Idiopathic pulmonary fibrosis: diagnosis, epidemiology and natural history. Respirology 2016;21(3):427-437. doi: 10.1111/resp.12683.

11. Oldham JM, Adegunsoye A, Valenzi E, et al. Characterisation of patients with interstitial pneumonia with autoimmune features. Eur Respir J 2016;47(6):1767-1775. doi: 10.1183/13993003.01565-2015.

12. Fischer A, Antoniou KM, Brown KK, et al.; ERS/ATS Task Force on Undifferentiated Forms of CTD-ILD. An official European Respiratory Society/American Thoracic Society research statement: interstitial pneumonia with autoimmune features. Eur Respir J 2015;46(4):976-987. doi: 10.1183/13993003.00150-2015.

\section{$\triangle$ Correspondencia:}

Dra. Ivette Buendía Roldán

Investigador en Ciencias Médicas "C", Instituto Nacional de Enfermedades Respiratorias Ismael Cosío Villegas.

Calzada de Tlalpan Núm. 4502,

Colonia Sección XVI, 14080, Ciudad de México.

Correo electrónico: ivettebu@yahoo.com.mx

Los autores declaran no tener conflicto de intereses. 